\title{
Protective Sliding Carbon-Based Nanolayers Prepared by Argon or Nitrogen Ion-Beam Assisted Deposition on Ti6Al4V Alloy
}

\author{
Petr Vlcak ${ }^{1}$ and Ivan Jirka ${ }^{2}$ \\ ${ }^{1}$ Faculty of Mechanical Engineering, Czech Technical University in Prague, Technicka 4, 16607 Prague, Czech Republic \\ ${ }^{2} J$. Heyrovsky Institute of Physical Chemistry, Academy of Sciences of the Czech Republic, v.v.i., Dolejskova 3, \\ 18223 Prague, Czech Republic
}

Correspondence should be addressed to Petr Vlcak; petr.vlcak@fs.cvut.cz

Received 22 September 2015; Revised 1 January 2016; Accepted 10 January 2016

Academic Editor: Waldeci P. Feio

Copyright (C) 2016 P. Vlcak and I. Jirka. This is an open access article distributed under the Creative Commons Attribution License, which permits unrestricted use, distribution, and reproduction in any medium, provided the original work is properly cited.

\begin{abstract}
The microstructure and the surface properties of samples coated by carbon-based nanolayer were investigated in an effort to increase the surface hardness and reduce the coefficient of friction of the Ti6Al4V alloy. Protective carbon-based nanolayers were fabricated by argon or nitrogen ion-beam assisted deposition at ion energy of $700 \mathrm{eV}$ on Ti6Al4V substrates. The Raman spectra indicated that nanolayers had a diamond-like carbon character with $\mathrm{sp}^{2}$ rich bonds. The $\mathrm{TiC}$ and $\mathrm{TiN}$ compounds formed in the surface area were detected by X-ray diffraction. Nanoscratch tests showed increased adhesion of a carbon-based nanolayer deposited with ion assistance in comparison with a carbon nanolayer deposited without ion assistance. The results showed that argon ion assistance leads to greater nanohardness than a sample coated by a carbon-based nanolayer with nitrogen ion assistance. A more than twofold increase in nanohardness and a more than fivefold decrease in the coefficient of friction were obtained for samples coated by a carbon-based nanolayer with ion assistance, in comparison with the reference sample.
\end{abstract}

\section{Introduction}

Carbon-based layers attract much attention due to their excellent sliding properties $[1,2]$ and their good wear resistance [3]. They could potentially be used as a protective film or as a functional layer in many industrial applications, for example, as protective thin films for recording media, sensors, and medical implants [4-6]. The deposition technique and the processing conditions have a direct influence on the chemical composition, the structure, and the physical, mechanical, and surface properties [7]. Various techniques have been used for depositing a carbon-based layer, including ion-beam assisted deposition (IBAD), chemical vapor deposition (CVD), plasma-enhanced chemical vapor deposition (PECVD), sputtering, and filtered cathodic vacuum arc deposition (FCVA) [8-12]. The IBAD method is very promising for the deposition of carbon-based layers. Ion bombardment significantly affects the performance of the layers. Preferred surface properties are produced by carbonbased coating surfaces with $\mathrm{sp}^{2}$ and $\mathrm{sp}^{3}$ hybridizations [13]. In order to optimize the carbon-based layer deposition method, it is necessary to understand the mechanisms involved in the growing process and in the degradation process $[13,14]$. In his review paper on amorphous carbon, Robertson presents his theory of the growth of amorphous carbon layers and introduces a model of subplantation [15]. Dopants such as nitrogen and hydrogen also play a critical role in respect to properties such as hardness, friction, and internal stress. Incorporating nitrogen in amorphous carbon increases the fraction of $\mathrm{sp}^{2}$ hybridized carbon atoms [16]. Thus carbonbased layers with nitrogen incorporation can provide lowfriction behavior, high wear resistance, and reduced internal stress [16-18]. In biomedical engineering, titanium alloys are used especially for their good biocompatibility and their low elastic modulus $[19,20]$. However, the surface properties of titanium-based implants, such as friction and wear resistance, are not fully satisfactory for use in biomedicine, and they are often modified.

In this work, the surface properties of Ti6Al4V titanium alloy were modified by ion-beam assisted deposition of 
carbon-based nanolayers. Protective sliding carbon-based nanolayers were deposited with the assistance of argon ions or nitrogen ions. The nanolayers were characterized by X-ray diffraction (XRD) and by Raman spectroscopy. The elemental chemical composition was measured by glow discharge optical emission spectroscopy (GD-OES). Nanohardness was measured by nanoindentation, adhesion was investigated by the nanoscratch test, and the sliding properties were tested on a ball-on-disc tribometer. The aim of our work was to investigate the effect of the deposition conditions on the microstructure and on the properties of carbon-based nanolayers, in order to improve the surface properties of biomedical Ti6Al4V alloy.

\section{Materials and Methods}

2.1. Preparation Process. Ti6Al4V titanium alloy with the $\alpha+$ $\beta$ phase microstructure was used as the substrate material. The disc samples (20 $\mathrm{mm}$ in diameter and $6 \mathrm{~mm}$ in thickness) were cut from a titanium alloy bar and were then ground with a series of waterproof abrasive papers up to 2000 grit. Final polishing was performed with $5 \mu \mathrm{m}$ and $1 \mu \mathrm{m}$ diamond paste. Before the deposition process, the samples were ultrasonically degreased in acetone and in isopropyl alcohol, both of them for 20 minutes, respectively.

The samples were placed on a sample holder mounted on a rotary manipulator in a vacuum chamber and were sputtered with $700 \mathrm{eV}$ argon ions to etch and activate the surface. A carbon nanolayer approximately $100 \mathrm{~nm}$ in thickness with a tungsten interlayer approximately $20 \mathrm{~nm}$ in thickness was deposited by electron beam evaporation of high purity carbon and tungsten targets. The deposition process was carried out with simultaneous assistance of argon or nitrogen ion bombardment. The deposition rate of the carbon and tungsten nanolayers was about $0.10 \mathrm{~nm} \cdot \mathrm{s}^{-1}$, and the work pressure was about $5 \cdot 10^{-3} \mathrm{~Pa}$. The energy of the accelerated argon ions and nitrogen ions was $700 \mathrm{eV}$, and the beam current density was about $80 \mu \mathrm{A} \cdot \mathrm{cm}^{-2}$.

2.2. Characterization Methods. The elemental chemical composition was measured by glow discharge optical emission spectroscopy (GD-OES). A LECO GDS850A optical emission spectrometer was used. GDS850A is an optical emission spectrometer with a Paschen-Runge polychromator with a focal length of $750 \mathrm{~mm}$, and it has a glow discharge spectral source with a flat cathode (Grimm-type). The sample itself serves as a cathode in this arrangement. Atomization of the sample surface is performed by the mechanism of cathode sputtering under an anomalous discharge. The process gas was argon. The spectrometer was calibrated using reference materials with a known composition and known sputter rates, and the "standard" calibration model was used.

The Raman spectra were measured using a Renishaw RM 1000 Raman microscope with Ar laser excitation at $514.5 \mathrm{~nm}$. The data were acquired from $3 \mu \mathrm{m}$ diameter spots with neutral filters limiting the laser power to $10 \%$, that is, around $0.1 \mathrm{~mW} \cdot \mu \mathrm{m}^{-2}$. This level of power was chosen after a set of measurements in which the power was slowly stepped up in a series of spectra acquisitions.
The phase composition was investigated by the X-ray diffraction (XRD) method. The measurements were performed on a PANalytical X'Pert PRO powder diffractometer. We chose a geometry with the parallel beam with an incident angle of $0.5^{\circ}$ with respect to the intensity of the diffraction pattern. The diffraction was recorded for the $2 \theta$ range $10^{\circ}-80^{\circ}$. Cobalt radiation with a wavelength of $0.1789 \mathrm{~nm}$ was used.

The nanohardness of the modified samples was investigated by nanoindentation testing. The dependence of the nanohardness on depth was registered. The measurements were performed on a Hysitron TriboIndenter ${ }^{\mathrm{Tm}}$ with a Berkovich diamond tip, using the CMX dynamic mode (continuous measurement of $\mathrm{X}$ ) with a maximum indentation force of $3000 \mu \mathrm{N}$ and a prescribed load/unload frequency of $85 \mathrm{~Hz}$. Nanoindentation testing proceeded at a temperature of $23.2^{\circ} \mathrm{C}$. Grids of $4 \times 4$ indents with $5 \mu \mathrm{m}$ separation were applied for each sample; see Figure 6. The tip area function was calibrated on the fused quartz sample according to a standard calibration procedure $[21,22]$.

The adhesion of carbon-based nanolayers was investigated by nanoscratch testing on the Hysitron TriboIndenter with scratch load resolution of $3 \cdot 10^{-3} \mu \mathrm{N}$, scratch force resolution of $0.5 \mu \mathrm{N}$, and displacement resolution of $3 \mathrm{~nm}$. The measurements were performed with a $60^{\circ}$ conical tip $<1 \mu \mathrm{m}$ in radius. The maximum load was up to $10000 \mu \mathrm{N}$ on a scratch length of $4500 \mathrm{~nm}$. The scratch scan recorded the penetration depth with increasing scratch distance, increasing scratch load, and increasing scratch force during nanoscratching.

A sliding test was performed in order to find out the coefficient of friction. A ball-on-disc tribometer was used. A constant normal load of 1 and $2 \mathrm{~N}$ was set during the test. The velocity was $8 \mathrm{~cm} \cdot \mathrm{s}^{-1}$. The second part of the sliding couple was a 100Cr6 steel ball $6 \mathrm{~mm}$ in diameter. The sliding tests proceeded at a laboratory ambient temperature of $23^{\circ} \mathrm{C}$, without lubrication and with relative humidity of $54 \%$. The measurements were performed without sucking off the abrasive wear products.

\section{Results and Discussion}

3.1. Elemental Chemical Composition. Typical GD-OES depth profiles of elements of the modified surface area are shown in Figure 1. The concentration profiles of the elements indicate that there is no sharp interface between the titanium alloy substrate, the tungsten interlayer, and the carbon-based nanolayer. The carbon-based nanolayer mixed with substrate atoms can be observed in both types of samples in Figures 1(a) and 1(b). The sample modified by a carbon-based nanolayer with argon ion assistance shows a larger area of atomic mixing. This indicates particularly the concentration profile of titanium, which was detected near the surface. Carbon and other elements detected at a deeper level can also indicate atomic mixing, but the concentration of these elements deep inside the substrate is influenced by the crater effect. A crater is formed during the GD-OES measurement, and sputtering from the crater wall influences the depth profiles. Diffusion may play some role, but the dominant influence is from sputtering from the crater wall. In the sample modified by a carbon-based nanolayer with 


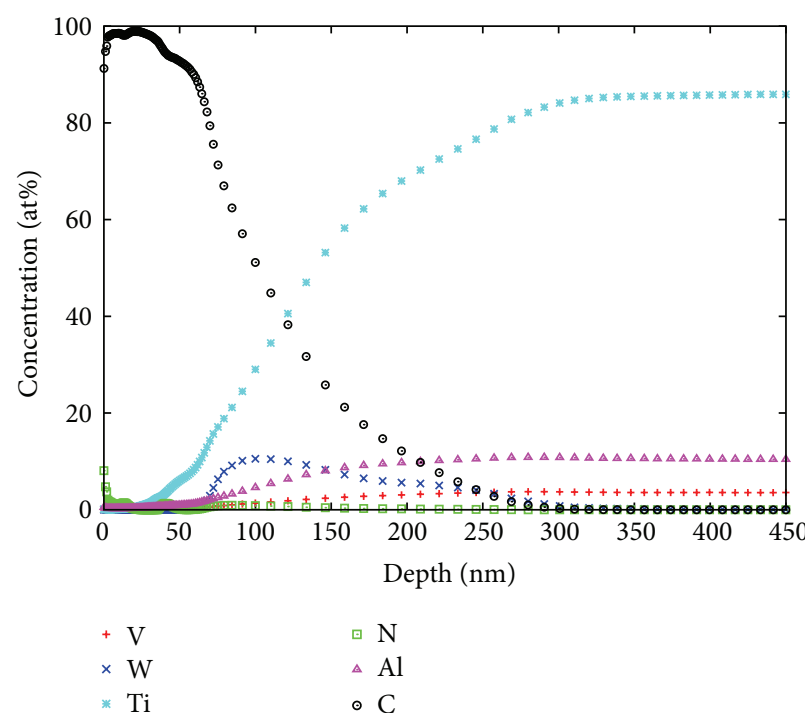

(a)

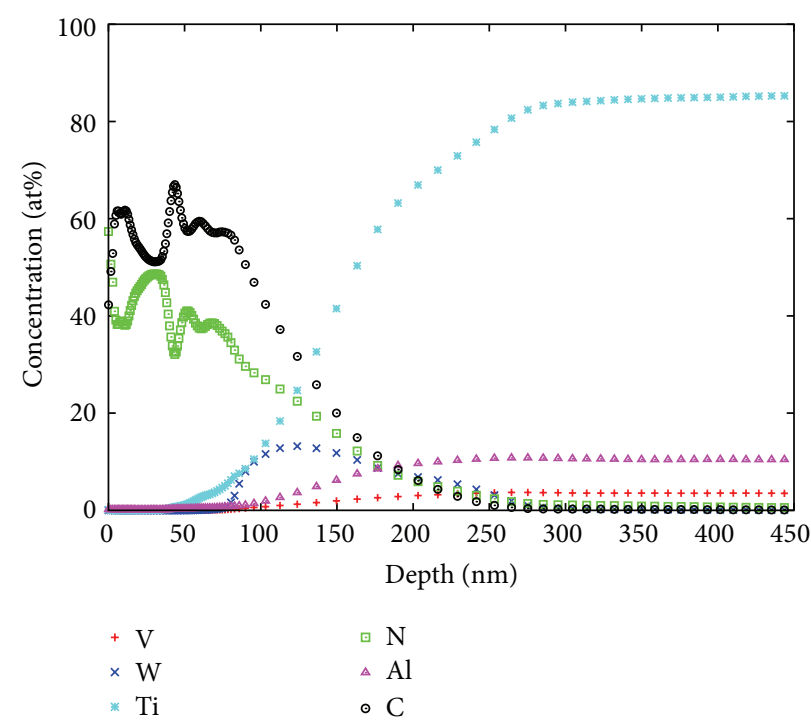

(b)

FIGURE 1: Elemental chemical composition of the surface area of the Ti6Al4V sample modified by a carbon-based nanolayer bombarded with argon ions (a) and the Ti6Al4V sample modified by a carbon-based nanolayer bombarded with nitrogen ions (b).

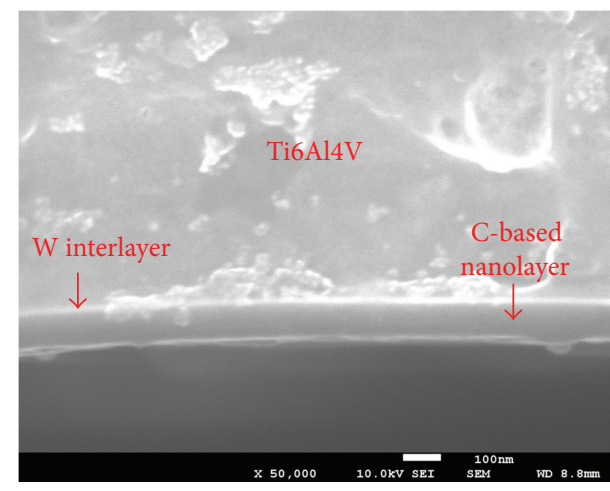

(a)

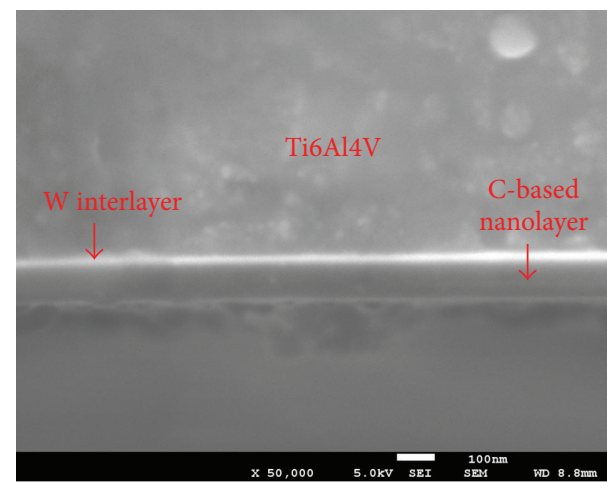

(b)

FIGURE 2: SEM micrograph of the polished transversal section of a sample modified by a carbon-based nanolayer with nitrogen ion assistance (a) and a sample modified by a carbon-based nanolayer with argon ion assistance (b).

nitrogen ion assistance, the average nitrogen concentration was found to be approximately 40 at $\%$ at a depth down to approximately $75 \mathrm{~nm}$. Nitrogen was also detected in the sample modified by a carbon-based nanolayer with argon ion assistance. The nitrogen on the surface of the sample and a shallow layer up to a few nanometers originated from contamination due to air exposure. Argon was used as the process gas, so the argon content cannot be evaluated from the GD-OES spectra.

3.2. Microstructure. During the IBAD process a continuous carbon-based monolayer was deposited on the Ti6Al4V alloy surface. Figure 2 presents SEM micrograph of the transversal section in which a continuous carbon-based layer with tungsten interlayer is shown. The continuous carbonbased monolayer was observed in all modified samples. SEM micrograph of the transversal section supplemented by EDS analysis (Figure 3) confirms the chemical composition of the created surface layer.

The microstructure and the phase composition of the modified samples were examined by XRD. This method can provide direct evidence of compounds formed in the modified surface area. An incident angle of $0.5^{\circ}$ was chosen with respect to the intensity of the diffraction spectra of a thin surface layer. Figure 4 shows the diffraction spectra of the modified samples. The alpha structural phase of the titanium alloy $(\alpha-\mathrm{Ti})$ was observed in all samples, while no minority beta structural phase $(\beta-\mathrm{Ti})$ was identified in the diffraction spectra. An additional $\alpha$-Ti phase with a larger lattice parameter appeared in the diffractograms of samples modified by a carbon-based nanolayer with nitrogen ion assistance. The implanted nitrogen ions were placed in the interstitial sites, and a solid solution of nitrogen was formed in the surface area. Some of the interstitial nitrogen atoms 


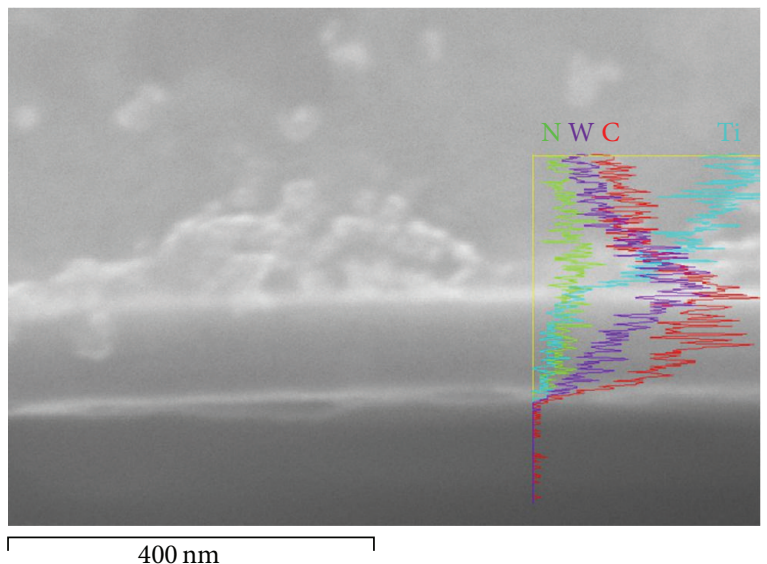

(a)

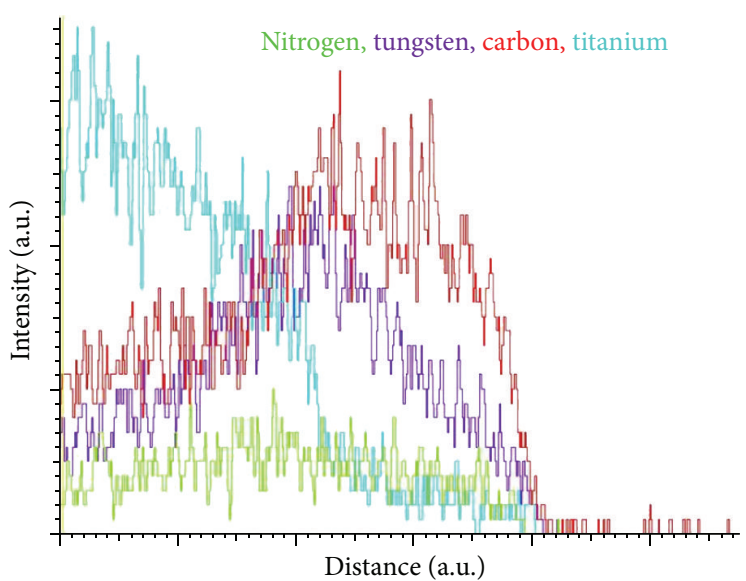

(b)

FIGURE 3: SEM image of the polished transversal section of a sample modified by a carbon-based nanolayer with nitrogen ion assistance and corresponding EDS line scan analysis.

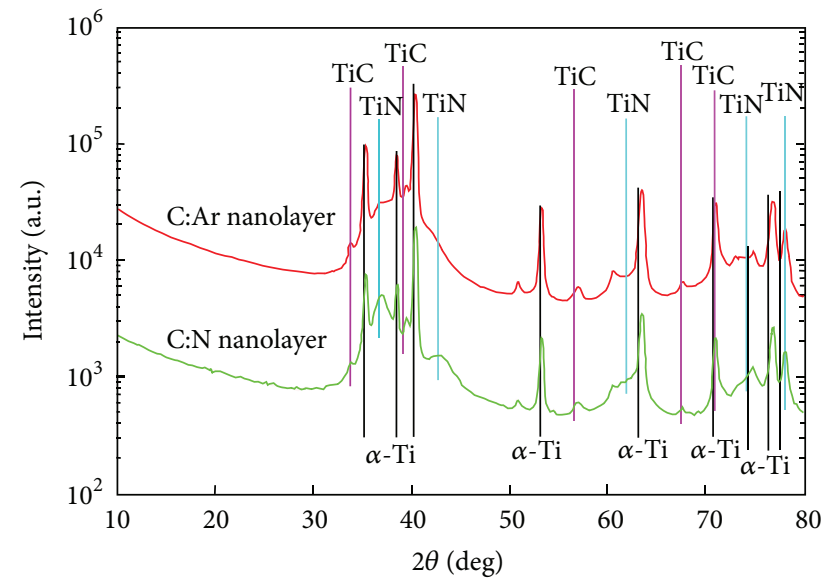

FIGURE 4: Diffraction spectra of samples coated by a carbon-based nanolayer with argon ion assistance (C:Ar nanolayer) and with nitrogen ion assistance (C:N nanolayer).

were arranged into the structure, and a TiN phase arose. These findings show that nitrogen ion assistance during the deposition of carbon-based nanolayers leads to the formation of a TiN compound on the interface of a carbon-based nanolayer/titanium alloy substrate and stabilizes the $\alpha$-Ti phase in the surface area. Schmidt et al. [23] and Vlcak et al. [24] have confirmed that nitrogen ion implantation on Ti$6 \mathrm{Al}-4 \mathrm{~V}$ stabilizes the $\alpha$-Ti phase and forms TiN compounds. In both types of samples, XRD analysis showed the presence of the TiC phase; see Figure 4. The results of XRD analysis confirmed that ion assistance during deposition of carbonbased nanolayers led to a mixed surface area in which TiN and $\mathrm{TiC}$ compounds were formed.

The bond structure of the carbon was investigated by Raman spectroscopy. Figure 5 compares the Raman spectra of a carbon-based nanolayer deposited with argon or nitrogen ion assistance and the spectrum of a carbon nanolayer deposited without ion assistance. The Raman

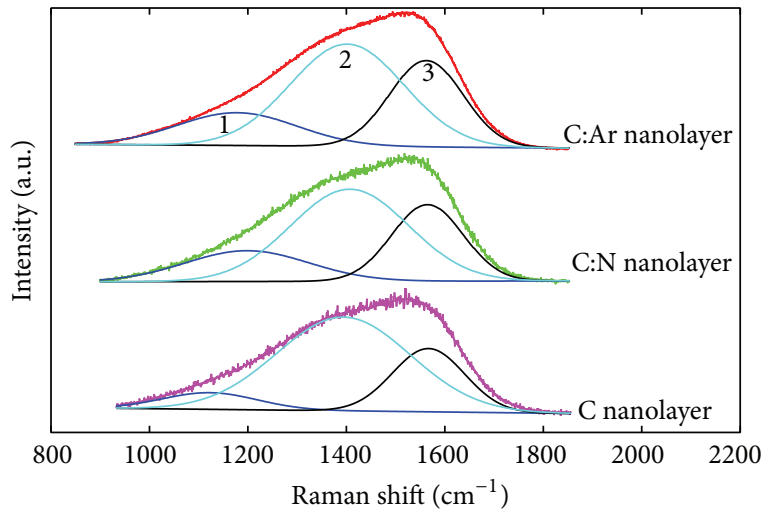

FIGURE 5: Raman spectra of a carbon-based nanolayer deposited with argon ion assistance (C:Ar), with nitrogen ion assistance (C:N), and without ion assistance $(\mathrm{C})$.

spectra in Figure 5 show one peak with a shoulder on the lower frequency. The position and the size of the peaks were determined by fitting to three Gaussian curves. The peak parameters are shown in Table 1 . The position of the characteristic peaks depends on the microstructure of the carbon-based nanolayer and the laser wavelength. Peak 3 at $\sim 1565 \mathrm{~cm}^{-1}$ is known as the G peak (graphite carbon), and peak 2 at $\sim 1400 \mathrm{~cm}^{-1}$ is known as the $\mathrm{D}$ peak (disordered graphitic carbon) [25]. The origin of peak 1 at $\sim 1180 \mathrm{~cm}^{-1}$ has been associated with the nanocrystalline or amorphous diamond fraction [26]. The data in Table 1 show that the ratio of the integrated areas under peaks 1 , 2 , and 3 decreases and the position of peak 3 (the G peak) moves slightly to lower frequencies due to ion assistance during the deposition process. The observed changes in the Raman spectra characterize the transformation of graphitic carbon into a DLC (diamond-like carbon) character, which explains the increase in hardness shown in Figure 7. Similar trends indicating the DLC character of the coatings were 
TABLE 1: Peak parameters of the fitted Raman spectra.

\begin{tabular}{lcccc}
\hline Sample & Peak 3 $\left(\mathrm{cm}^{-1}\right)$ & Peak $2\left(\mathrm{~cm}^{-1}\right)$ & Peak 1 $\left(\mathrm{cm}^{-1}\right)$ & Integrated areas ratio $(1+2) / 3$ \\
\hline C:Ar & 1563 & 1402 & 1178 & 2.43 \\
C:N & 1565 & 1407 & 1198 & 2.67 \\
C & 1567 & 1394 & 1123 & 3.09 \\
\hline
\end{tabular}

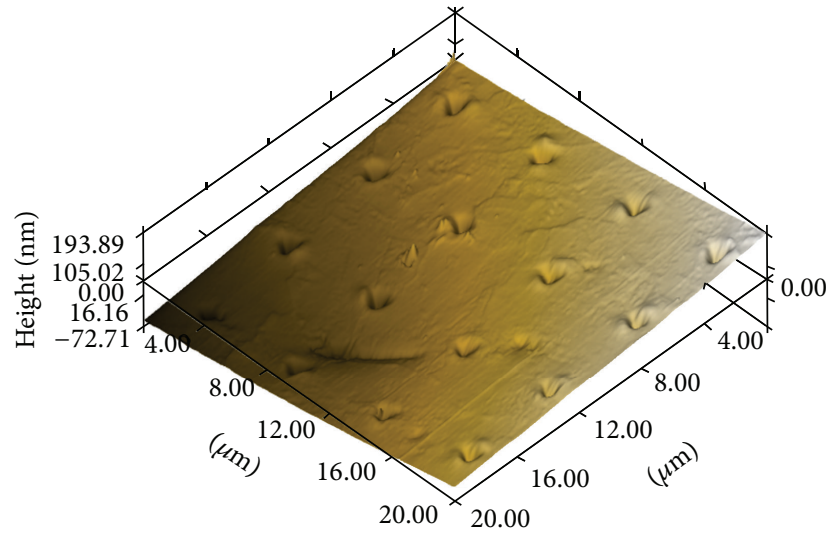

(a)

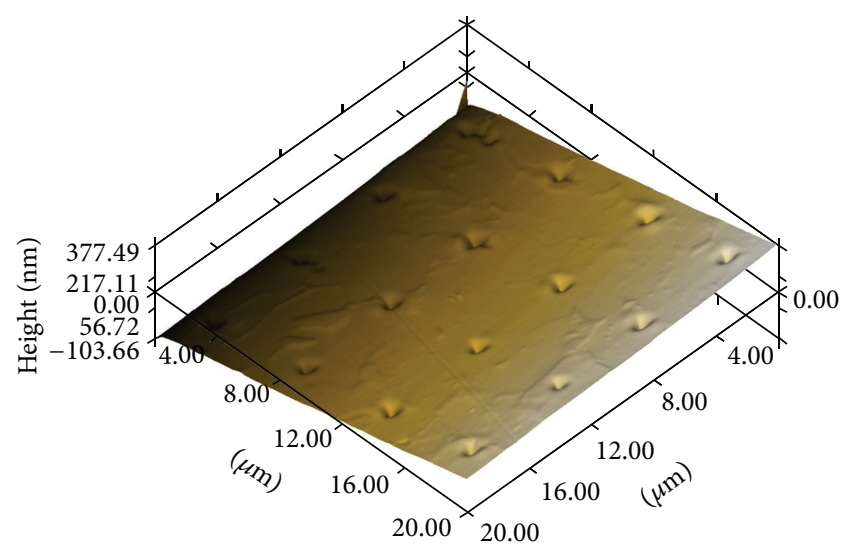

(b)

FIGURE 6: Representative 3D post scans of indents after nanoindentation testing of a sample modified by a carbon-based nanolayer with argon ion assistance (a) and a sample modified by a carbon-based nanolayer with nitrogen ion assistance (b).

observed by Morshed et al. [26] and by Ziebert et al. [27]. The correlation with the nanohardness results shows that the nanohardness increases as the ratio of the integrated areas decreases. It can be estimated from the Raman spectra that the carbon-based nanolayer consists predominantly of $\mathrm{sp}^{2}$ bonds with a small $\mathrm{sp}^{3}$ content [15].

3.3. Nanohardness. The nanohardness of the modified samples was investigated by nanoindentation testing. Representative post scans of the indents are shown in Figure 6. A small plastic deformation region can be seen around the indents. The resulting nanohardness depth profiles are shown in Figure 7. It is seen that the nanohardness at shallow depths up to few nanometers is small, and it increases rapidly with contact depth to the maximum value. The nanohardness values in this region are influenced by the error originating from the standard calibration of the tip area function on fused quartz $[21,22]$. As the contact depth increases further, the nanohardness decreases from the maximum value and converges to the hardness of the reference sample (see Figure 7). The reduction in nanohardness is a consequence of the influence of the softer titanium alloy substrate. The maximum indentation hardness values of the modified samples are shown in Table 2. The nanohardness of samples coated by a carbon-based nanolayer with argon ion assistance was increased by a factor of approximately 2.31, and the nanohardness of a sample coated by a carbonbased nanolayer with nitrogen ion assistance was increased by a factor of approximately 2.22 , compared to the maximum hardness value of the reference sample. The increase in nanohardness can be attributed mainly to the bond structure of carbon. It can be assumed that the mixing area may also have an impact on the increase in nanohardness. The hard $\mathrm{TiC}$ and TiN compounds detected by XRD in the interface of the carbon-based nanolayer/titanium alloy substrate also hardened the surface area. The nanohardness depth profiles in Figure 7 contain the depth profile of the nanohardness of the sample coated by a carbon nanolayer without ion assistance. The maximum hardness value of the sample coated with a $100 \mathrm{~nm}$ thick carbon nanolayer without ion assistance is $8.74 \pm 0.64 \mathrm{GPa}$ at a depth of $30 \mathrm{~nm}$. The nanohardness of the reference sample decreases with increasing contact depth from a maximum value of $5.33 \pm 0.41 \mathrm{GPa}$ to an almost constant value of $4.6 \mathrm{GPa}$. The nonconstant course of the nanohardness of the reference sample may be caused by mechanical strengthening of the surface due to cutting and polishing.

3.4. Adhesion. The adhesion of nanolayers to a titanium alloy substrate was investigated by nanoscratch testing over a continuous range of scratch loads. The trend of the penetration depth according to the scratch load for samples coated by carbon-based nanolayers with ion assistance and for the sample coated by a carbon nanolayer without ion assistance is presented in Figure 8. The penetration depth increases with increasing scratch load. A comparison of the curves in Figure 8 shows that the penetration depth for the same scratch load value is greater for the sample coated by a carbon nanolayer without ion assistance. This shows that the resistance to penetration of the scratch tip increases due to 
TABLE 2: Maximum nanohardness values of the modified samples and the reference sample.

\begin{tabular}{lccc}
\hline Sample & C:Ar & C:N & Ref. sample \\
\hline Maximum indentation hardness $(\mathrm{GPa})$ & $12.34 \pm 0.64$ & $11.84 \pm 0.34$ & $5.33 \pm 0.41$ \\
Contact depth $(\mathrm{nm})$ & 11 & 14 & 6 \\
\hline
\end{tabular}

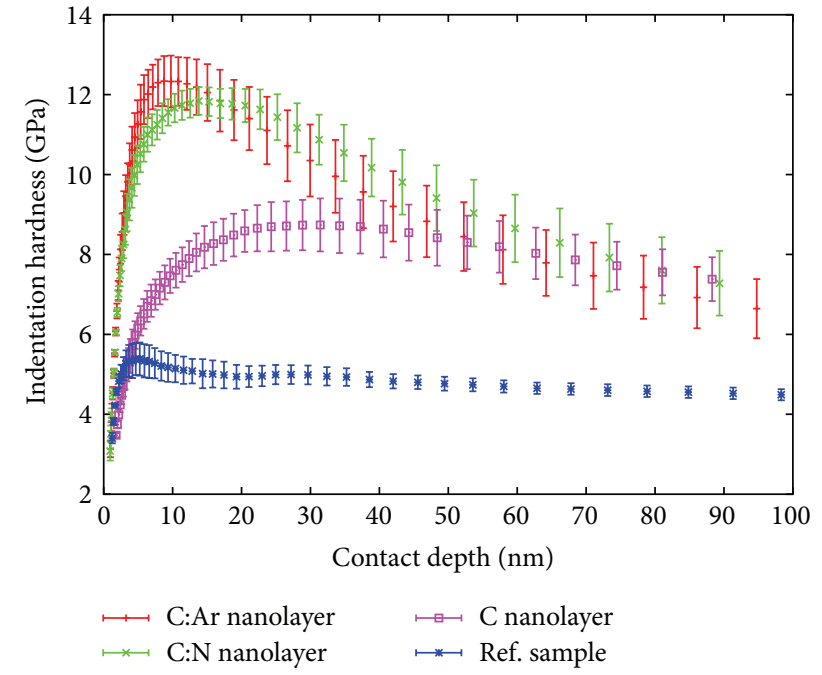

FIGURE 7: Resulting nanohardness depth profiles.

ion assistance during the nanolayer deposition process. This finding is in agreement with the nanohardness results. The sample coated by a carbon-based nanolayer with argon ion assistance and sample coated by a carbon-based nanolayer with nitrogen ion assistance show similar trends. A small difference can be observed for a greater scratch load from approximately $6000 \mu \mathrm{N}$. Figure 8 shows a smooth course of the penetration depth along the scratch load for samples coated by a carbon-based nanolayer with ion assistance, while the course of the penetration depth of the sample coated by a carbon nanolayer without ion assistance shows step increases in the penetration depth at a constant scratch load. Similar trends can be observed in Figure 9, which presents the scratch force versus the scratch distance. The visible jump of the scratch force curve for the sample coated by a carbon nanolayer without ion assistance represents the increase in the scratch distance without an increase in the scratch force. The jump indicates delamination of the carbon nanolayer, which is confirmed by the slight decrease in the scratch force in Figure 9 and is accompanied by the step increase in the penetration depth shown in Figure 8. The carbon-based nanolayers deposited with ion assistance show good adhesion with no signs of delamination of the nanolayers throughout the course of the nanoscratch tests. It can be assumed that the mixing area detected by GDOS and XRD improved the adhesion of the nanolayers.

3.5. Sliding Behaviour. Figure 10 shows the evolution of the coefficient of friction at a normal load of $1 \mathrm{~N}$ for samples coated by carbon-based nanolayers with ion assistance,

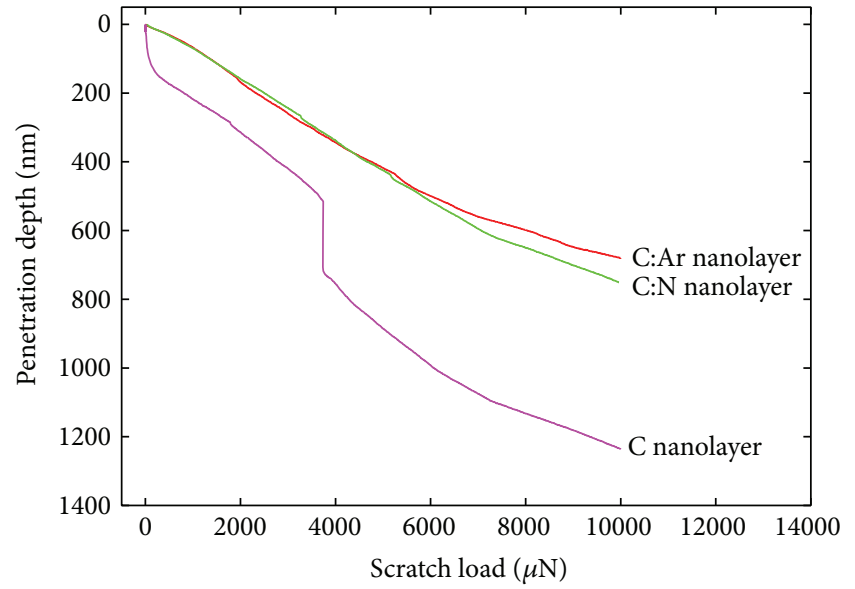

FIgURE 8: The trend of the penetration depth according to the scratch load for samples coated by carbon-based nanolayers with ion assistance and the sample coated by a carbon nanolayer without ion assistance.

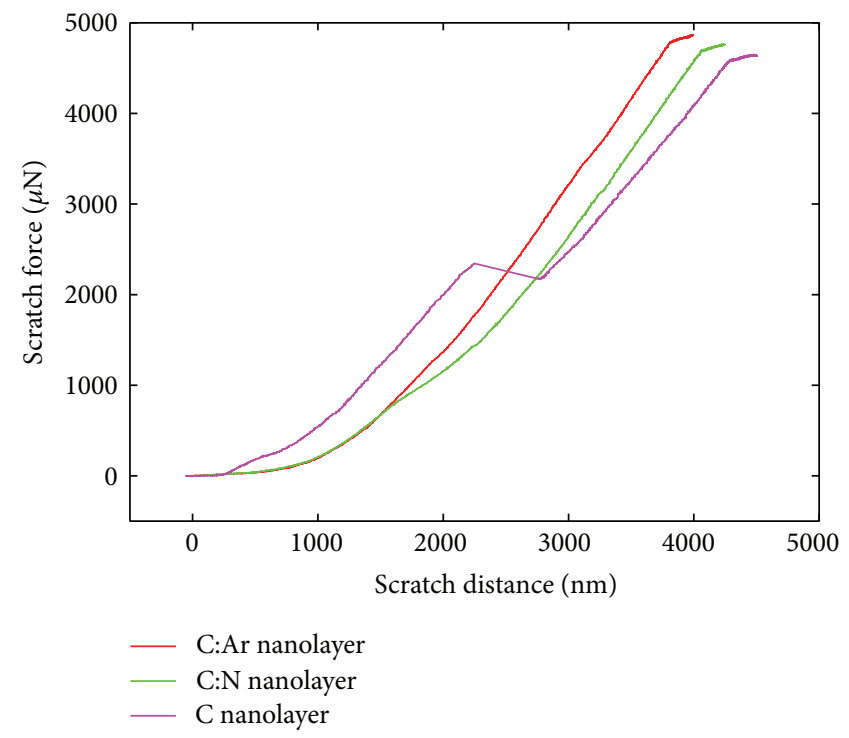

FIGURE 9: Scratch force versus scratch distance during nanoscratch experiments.

without ion assistance, and for the reference sample as a function of the number of cycles. The friction curve of the sample coated by a carbon nanolayer without ion assistance can be divided into two parts. The first part represents the low-friction regime, while the second part represents the increased friction regime. The friction coefficient in the low-friction part is low, having a value of about 0.13 . The 


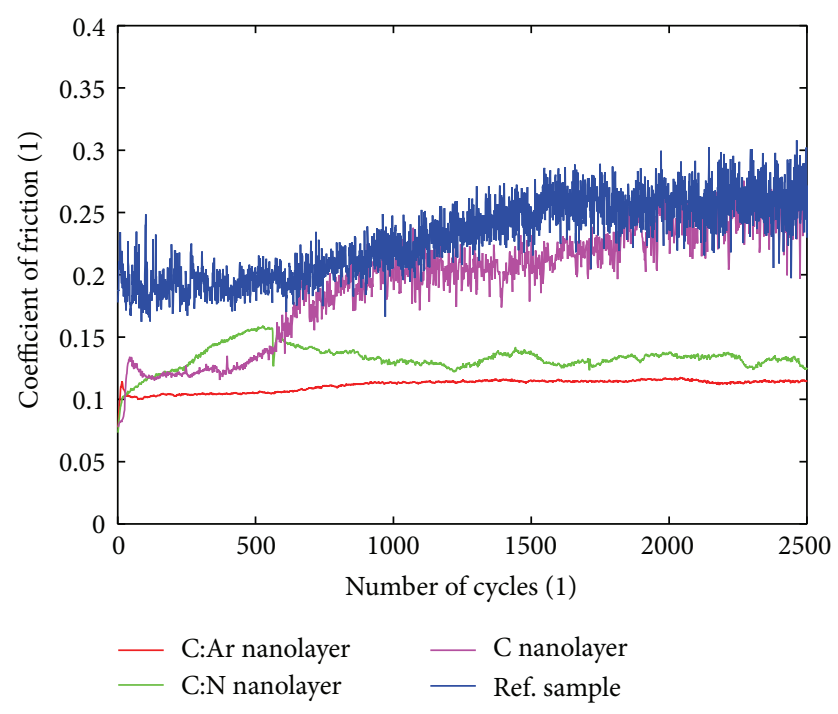

FIgURE 10: A comparison of the friction coefficient as a function of the number of cycles for samples coated by a carbon-based nanolayer with ion assistance, without ion assistance, and for the reference Ti6Al4V sample (ball-on-disc test, $1 \mathrm{~N}$ normal load, $100 \mathrm{Cr} 6 \mathrm{ball}, 8.00 \mathrm{~cm} \cdot \mathrm{s}^{-1}$ linear speed).

low-friction coefficient value remained constant until the carbon nanolayer was destroyed. The part with increased friction is characterized by a sharp increase in the coefficient of friction at approximately 600 cycles. In this part, the friction coefficient ranges from 0.17 to 0.25 , which is the level of the friction coefficient of the reference sample; see Figure 10. The samples coated by carbon-based nanolayers with ion assistance showed a low-friction coefficient, which was almost constant until the end of the friction test. The sample coated by a carbon-based nanolayer with argon ion assistance showed a coefficient of friction of approximately 0.11 , and the sample coated by a carbon-based nanolayer with nitrogen ion assistance showed a coefficient of friction of approximately 0.13 . This means an approximately twofold decrease in the friction coefficient for samples coated by carbon-based nanolayers with ion assistance in comparison with the reference sample, which had a friction coefficient of approximately 0.25 . Figure 10 shows that the friction curve of a sample coated by a carbon-based nanolayer with argon ion assistance was very stable, without fluctuation. Figure 11 compares the friction curves of samples coated by carbonbased nanolayers with ion assistance and the reference sample at a normal load of $2 \mathrm{~N}$. The friction coefficient between the sample coated by a carbon-based nanolayer with argon ion assistance and the sample coated by a carbon-based nanolayer with nitrogen ion assistance stabilized at the same value of 0.11 . This friction coefficient is comparable with the friction coefficient obtained at a normal load of $1 \mathrm{~N}$, while the friction coefficient of the reference sample was increased more than twofold to a value of 0.6 . This means a greater than fivefold decrease in the friction coefficient for samples coated by a carbon-based nanolayer with ion assistance, in comparison with the reference sample. The resulting reduction in the friction coefficient is attributed to the sliding properties of

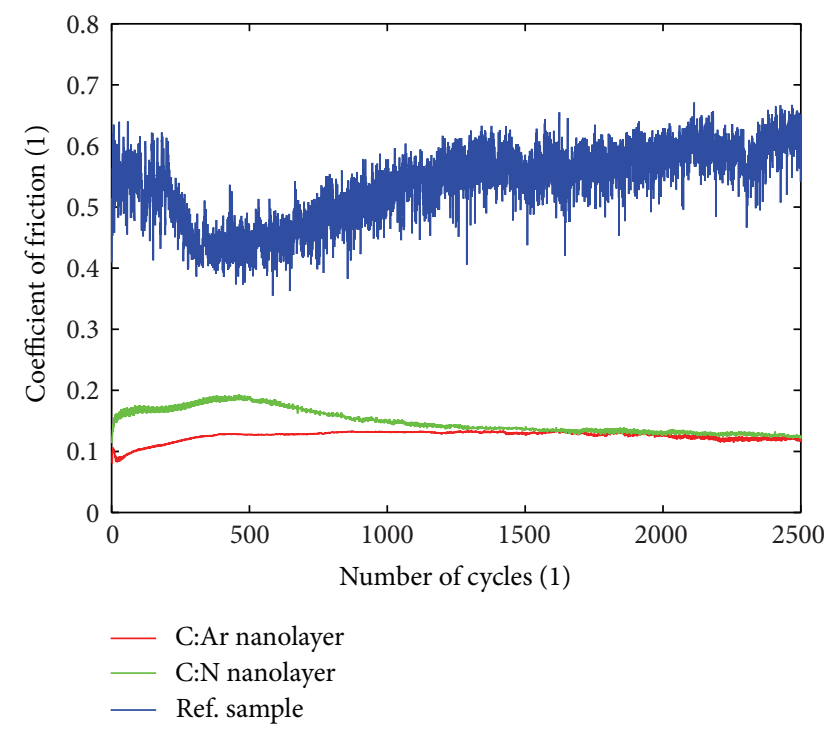

FIGURE 11: Coefficient of friction of samples coated by a carbonbased nanolayer with ion assistance and the reference Ti6Al4V sample (ball-on-disc test, $2 \mathrm{~N}$ normal load, $100 \mathrm{Cr} 6 \mathrm{ball}, 8.00 \mathrm{~cm} \cdot \mathrm{s}^{-1}$ linear speed).

a carbon-based nanolayer deposited with ion assistance. It is interesting that no effect of the species of ions on the friction coefficient value was observed. The hard $\mathrm{TiC}$ and TiN compounds detected by XRD in the titanium matrix hardened the surface area after ion-beam assisted deposition of a carbon-based nanolayer, as shown by the depth profiles of the indentation hardness. The adhesion-enhanced carbonbased nanolayers on the hardened surface acted as protective nanolayers with a lubricating effect.

The surface morphology of modified samples was characterized by atomic force microscopy (AFM). The modified surface is very smooth as follows from AFM scans in Figure 12. The root mean square (RMS) roughness of all modified samples ranges between 3.56 and $6.84 \mathrm{~nm}$. The characteristic AFM images of the sliding wear scars after sliding at a normal load of $2 \mathrm{~N}$ with their corresponding surface roughness profiles are presented in Figure 12. Line scan profiles show that the maximum deep of wear track of the samples coated by a carbon-based nanolayer with argon ion assistance or with nitrogen ion assistance is approximately $60 \mathrm{~nm}$. The same character of wear can be caused by lowfriction coefficient, higher hardness, and low value of surface roughness that originate from amorphous DLC character of carbon-based nanolayer.

\section{Conclusion}

Protective carbon-based nanolayers were fabricated by argon or nitrogen ion-beam assisted deposition at ion energy of $700 \mathrm{eV}$ on Ti6Al4V substrates. The carbon-based nanolayers were found to have a diamond-like carbon character with $\mathrm{sp}^{2}$ rich bonds. TiC and TiN compounds were found in the surface area. Increased adhesion of carbon-based nanolayers deposited with ion assistance was observed, in comparison 

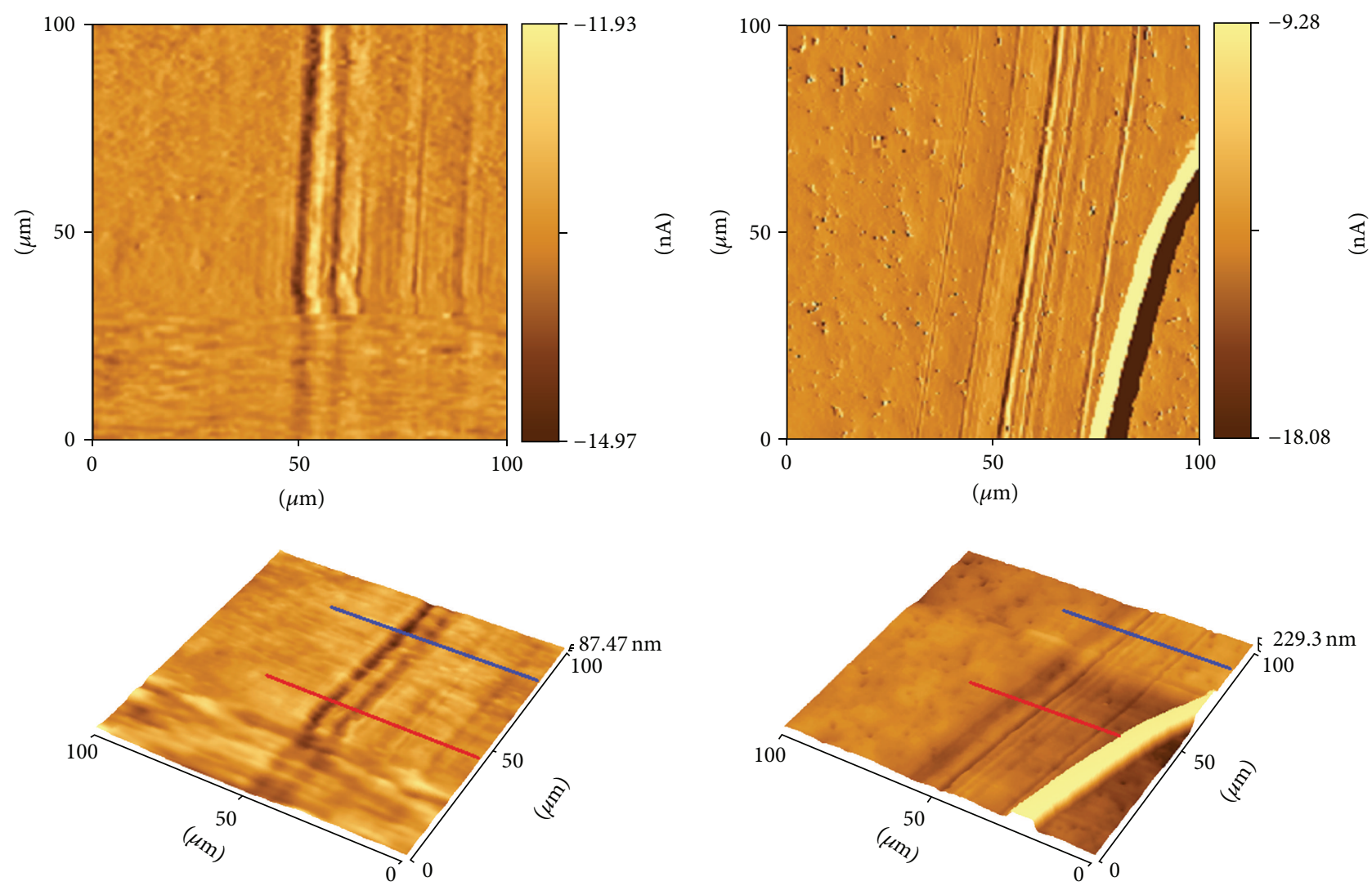

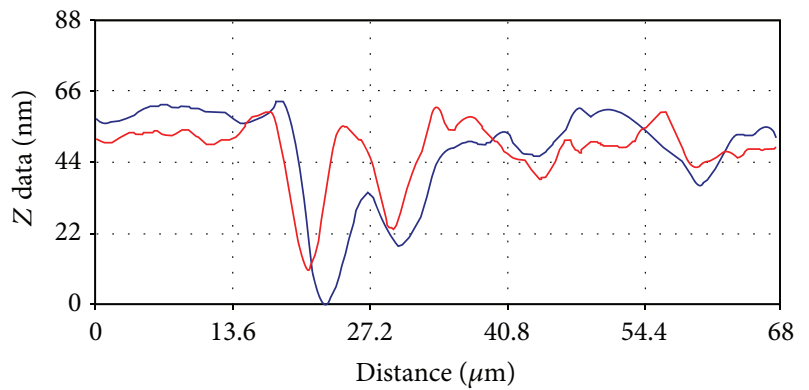

(a)

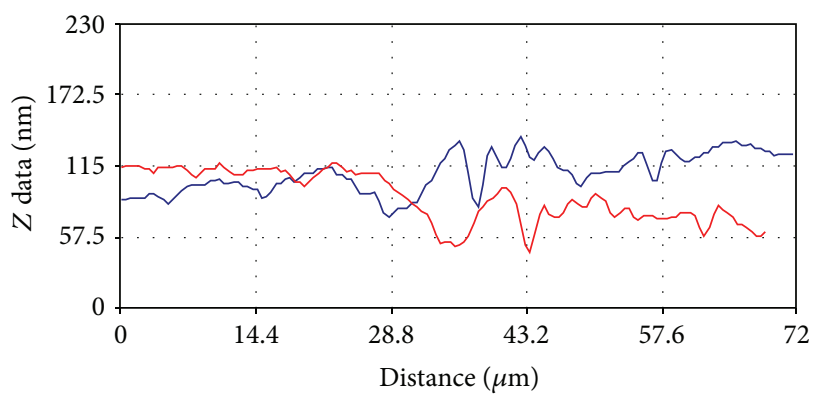

(b)

FIGURE 12: AFM images with the corresponding surface profiles of a sample modified by a carbon-based nanolayer with argon ion assistance (a) and a sample modified by a carbon-based nanolayer with nitrogen ion assistance (b).

with carbon nanolayers deposited without ion assistance. The nanohardness of a sample coated by a carbon-based nanolayer with argon ion assistance was increased by a factor of 2.31, and the nanohardness of a sample coated by a carbon-based nanolayer with nitrogen ion assistance was increased by a factor of 2.22, in comparison with the reference sample. The very stable course and the same coefficient of friction value of 0.11 of the samples coated by a carbonbased nanolayer with argon ion assistance or with nitrogen ion assistance were maintained at a normal load of $2 \mathrm{~N}$. In comparison with the reference sample, a greater than fivefold decrease in the coefficient of friction at a normal load of $2 \mathrm{~N}$ for samples coated by carbon-based nanolayers with ion assistance was obtained.

\section{Conflict of Interests}

The authors declare that there is no conflict of interests regarding the publication of this paper.

\section{Acknowledgments}

All the research support provided by the Faculty of Mechanical Engineering of the Czech Technical University in Prague (FME of CTU in Prague) and by the staff of the faculty is gratefully acknowledged. In addition, the authors would like to thank colleagues (Josef Sepitka and Zdenek Tolde) at FME of CTU in Prague for their help with nanomechanical and friction testing, and they express special thanks to Ivan 
Gregora at the Institute of Physics, Academy of Sciences of the Czech Republic, for his friendly help with the Raman spectroscopy measurements. This research has been supported by Ministry of Education, Youth and Sports of the Czech Republic Project SGS15/186/OHK2/3T/12 of the Czech Technical University in Prague.

\section{References}

[1] X. Yu, Y. Qin, C. B. Wang, Y. Q. Yang, and X. C. Ma, "Effects of nanocrystalline silver incorporation on sliding tribological properties of Ag-containing diamond-like carbon films in multi-ion beam assisted deposition," Vacuum, vol. 89, no. 1, pp. 82-85, 2013.

[2] S. Miyake, T. Shindo, and M. Miyake, "Regression analysis of the effect of bias voltage on nano- and macrotribological properties of diamond-like carbon films deposited by a filtered cathodic vacuum arc ion-plating method," Journal of Nanomaterials, vol. 2014, Article ID 657619, 13 pages, 2014.

[3] T. Hayashi, A. Matsumuro, M. Muramatsu, M. Kohzaki, and K. Yamaguchi, "Wear resistance of carbon nitride thin films formed by ion beam assisted deposition," Thin Solid Films, vol. 376, no. 1-2, pp. 152-158, 2000.

[4] T. M. Chao and A. H. Tan, "DLC deposition parameters optimization for head disk design interface with a thermal protrusion slider from tribological point of view," Materials and Design, vol. 48, pp. 58-67, 2013.

[5] B. Wu, Z. Ou, X. Ju, and S. Hou, "Carbon nanotubes/gold nanoparticles composite film for the construction of a novel amperometric choline biosensor," Journal of Nanomaterials, vol. 2011, Article ID 464919, 6 pages, 2011.

[6] Y.-L. Pei and Y. Luan, "Surface modification of NiTi alloys using nitrogen doped diamond-like carbon coating fabricated by plasma immersion ion implantation and deposition," Journal of Alloys and Compounds, vol. 581, pp. 873-876, 2013.

[7] V. J. Trava-Airoldi, L. F. Bonetti, G. Capote, L. V. Santos, and E. J. Corat, "A comparison of DLC film properties obtained by r.f. PACVD, IBAD, and enhanced pulsed-DC PACVD," Surface and Coatings Technology, vol. 202, no. 3, pp. 549-554, 2007.

[8] H. Huang, X. Wang, and J. He, "Synthesis and properties of graphite-like carbon by ion beam-assisted deposition," Materials Letters, vol. 57, no. 22-23, pp. 3431-3436, 2003.

[9] J. P. Davim and F. Mata, "Chemical vapour deposition (CVD) diamond coated tools performance in machining of PEEK composites," Materials and Design, vol. 29, no. 8, pp. 1568-1574, 2008.

[10] S. A. Ahmad Kamal, R. Ritikos, and S. Abdul Rahman, "Wetting behaviour of carbon nitride nanostructures grown by plasma enhanced chemical vapour deposition technique," Applied Surface Science, vol. 328, pp. 146-153, 2015.

[11] P. J. Kelly, R. D. Arnell, W. Ahmed, and A. Afzal, "Novel engineering coatings produced by closed-field unbalanced magnetron sputtering," Materials and Design, vol. 17, no. 4, pp. 215-219, 1996.

[12] G.-G. Wang, H.-Y. Zhang, W.-Y. Li et al., "The preparation and evaluation of graded multilayer ta-C films deposited by FCVA method," Applied Surface Science, vol. 257, no. 11, pp. 5064-5069, 2011.

[13] A. Markwitz, B. Mohr, and J. Leveneur, "Room temperature diamond-like carbon coatings produced by low energy ion implantation," Nuclear Instruments and Methods in Physics
Research, Section B: Beam Interactions with Materials and Atoms, vol. 331, pp. 144-148, 2014.

[14] W. Yue, X. Gao, C. Wang, Z. Fu, X. Yu, and J. Liu, "Microstructure and friction-reducing performance of sulfurized $\mathrm{W}$ doped diamond-like carbon film," Materials Letters, vol. 73, pp. $202-$ 205, 2012.

[15] J. Robertson, "Diamond-like amorphous carbon," Materials Science and Engineering: R: Reports, vol. 37, no. 4-6, pp. 129-281, 2002.

[16] D. F. Wang, K. Kato, and N. Umehara, "Mechanical characterization and tribological evaluation of ion-beam-assisted sputter coatings of carbon with nitrogen incorporation," Surface and Coatings Technology, vol. 123, no. 2-3, pp. 177-184, 2000.

[17] A. Grill, "Tribology of diamondlike carbon and related materials: an updated review," Surface and Coatings Technology, vol. 94-95, pp. 507-513, 1997.

[18] P. Vlcak, F. Cerny, Z. Weiss et al., “The effect of nitrogen ion implantation on the surface properties of Ti6Al4V alloy coated by a carbon nanolayer," Journal of Nanomaterials, vol. 2013, Article ID 475758, 8 pages, 2013.

[19] S. Podzimek, M. Tomka, T. Nemeth, L. Himmlova, P. Matucha, and J. Prochazkova, "Influence of metals on cytokines production in connection with successful implantation therapy in dentistry," Neuroendocrinology Letters, vol. 31, no. 5, pp. 657$662,2010$.

[20] J. Procházková, Š. Podzimek, M. Tomka et al., "Metal alloys in the oral cavity as a cause of oral discomfort in sensitive patients," Neuroendocrinology Letters, vol. 27, no. 1, pp. 53-58, 2006.

[21] W. C. Oliver and G. M. Pharr, "An improved technique for determining hardness and elastic modulus using load and displacement sensing indentation experiments," Journal of Materials Research, vol. 7, no. 6, pp. 1564-1583, 1992.

[22] K. Rokosz, T. Hryniewicz, J. Lukeš, and J. Šepitka, "Nanoindentation studies and modeling of surface layers on austenitic stainless steels by extreme electrochemical treatments," Surface and Interface Analysis, vol. 47, no. 6, pp. 643-647, 2015.

[23] H. Schmidt, A. Schminke, M. Schmiedgen, and B. Baretzky, "Compound formation and abrasion resistance of ionimplanted Ti6Al4V," Acta Materialia, vol. 49, no. 3, pp. 487-495, 2001.

[24] P. Vlcak, F. Cerny, J. Drahokoupil, J. Sepitka, and Z. Tolde, "The microstructure and surface hardness of Ti6Al4V alloy implanted with nitrogen ions at an elevated temperature," Journal of Alloys and Compounds, vol. 620, pp. 48-54, 2015.

[25] Y. Funada, K. Awazu, K. Shimamura, and M. Iwaki, "Thermal properties of DLC thin films bombarded with ion beams," Surface and Coatings Technology, vol. 103-104, pp. 389-394, 1998.

[26] M. M. Morshed, B. P. McNamara, D. C. Cameron, and M. S. J. Hashmi, "Stress and adhesion in DLC coatings on 316L stainless steel deposited by a neutral beam source," Journal of Materials Processing Technology, vol. 143-144, no. 1, pp. 922-926, 2003.

[27] C. Ziebert, M. Rinke, M. Stüber, S. Ulrich, and H. Holleck, "Interfaces and temperature stability of stepwise graded DLC films studied by nanoindentation and Raman spectroscopy," Surface and Coatings Technology, vol. 200, no. 1-4, pp. 1127-1131, 2005. 

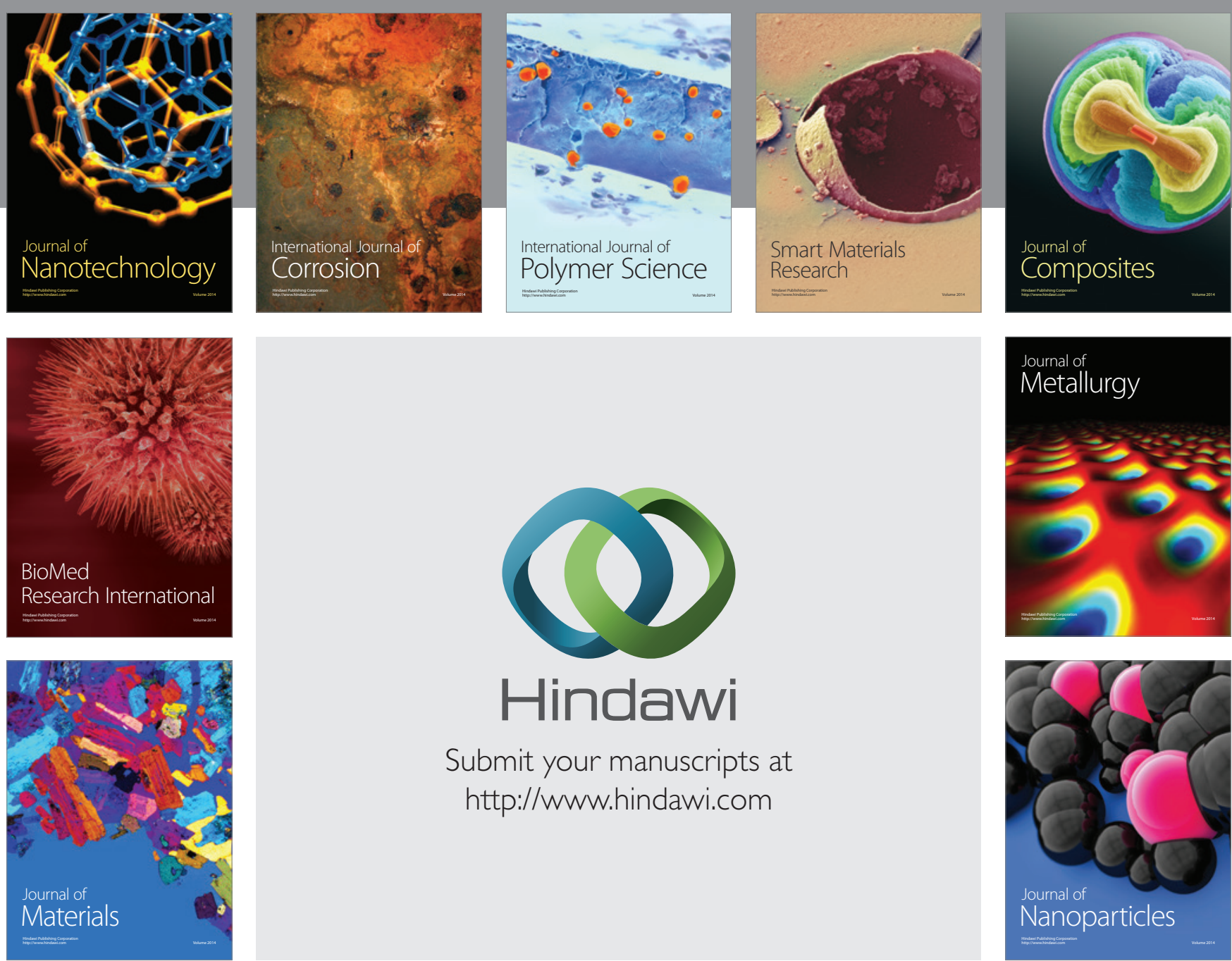

\section{Hindawi}

Submit your manuscripts at

http://www.hindawi.com

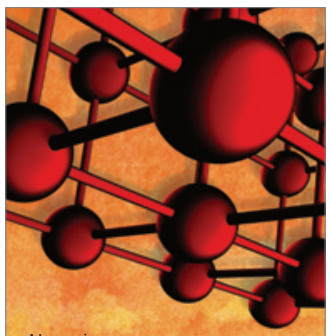

Materials Science and Engineering
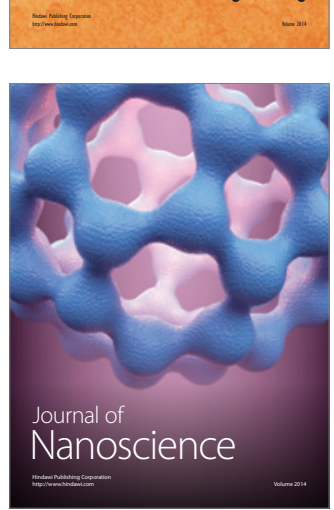
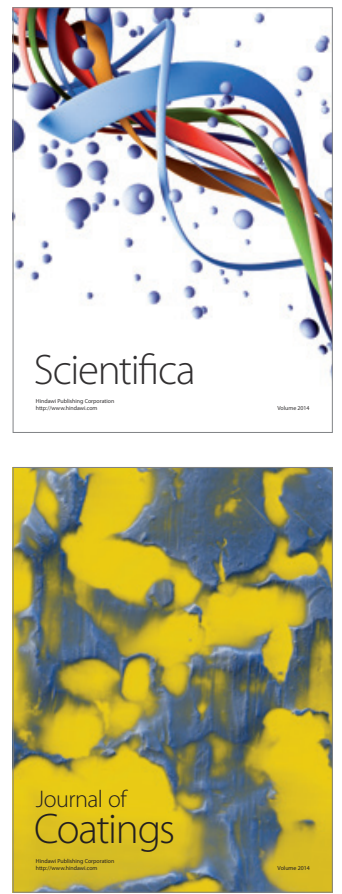
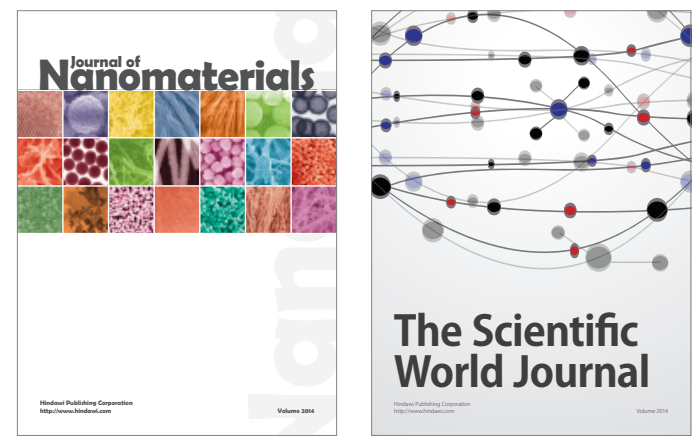

The Scientific World Journal
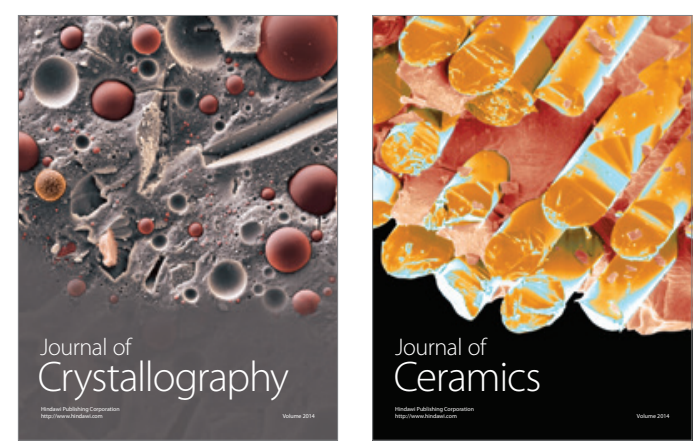
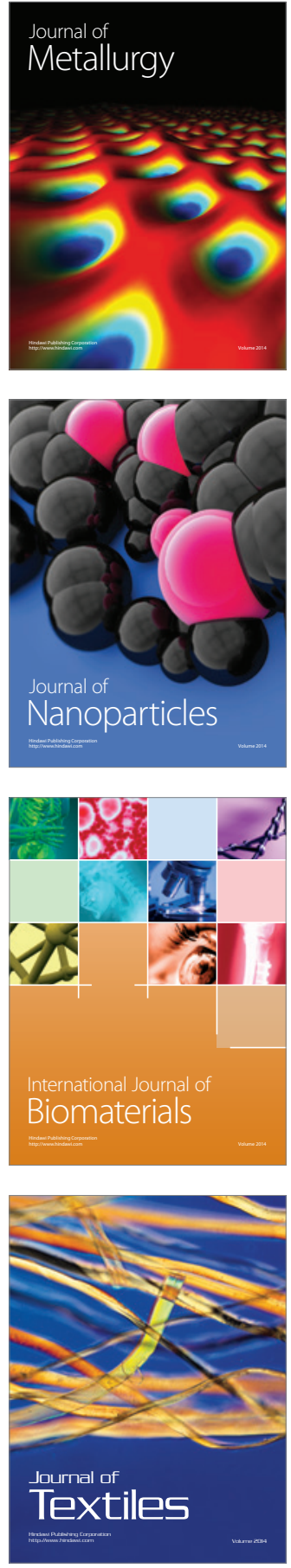